\title{
Heuristic Algorithm for Velocity Scheduling with a Schönflies-Motion Generator
}

\author{
Bruno Belzile and Jorge Angeles \\ Department of Mechanical Engineering \& Centre for Intelligent Machines, McGill University, \\ Canada \\ $\{$ bruno, angeles\}@cim.mcgill.ca
}

\begin{abstract}
The authors propose a trajectory-planning algorithm to minimize the maximum torque required to produce a prescribed trajectory with a pick-andplace robot. This is done with a scheduling of the velocity of the moving plate, while following the Adept path. Simulation tests are conducted with the dynamics model of an isostatic Schönflies-motion generator, but the proposed algorithm can be applied to any architecture capable of producing Schönflies motions. Results show a reduction of more than $40 \%$ of the maximum torque required from the actuators, regardless of the cycle time, when traversing the path at $1 \mathrm{~Hz}$ and higher.
\end{abstract}

Keywords: Kinematics, dynamics, pick-and-place, Schönflies-motion generators

\section{Introduction}

Pick-and-place operations (PPO) appear in several tasks such as packaging, moving an object from one workstation to another, etc. To produce these operations, industry asks for robots capable of fast PPO while keeping an accurate trajectory-tracking. Schönflies motion generators (SMG), machines capable of three translations and one rotation about an axis of fixed direction (generally vertical), are commonly used [1]. While serial architectures exist and are well known, such as the Selective Compliance Articulated Robot Arm (SCARA) [2], nowadays hybrid and parallel architectures are generally chosen because of their lower moving mass, enabling them to reach higher speed. A wide range of these Parallel Kinematic Machines (PKM) has been recorded in the literature [3-7].

In order to meet the industry demands for ever faster pick-and-place robots, trajectory planning and cycle time play a crucial role. While the time a robot takes to move its moving plate (MP) from one pose to another is critical, what happens in-between is less important. In several papers, the optimization of the trajectory with different objective functions, many of them focusing on the prescribed path, has been reported. For instance, researchers have used the dynamic response of cable robots to find the optimum feasible path [8-10]. Others have focused on the optimization of the motion profile. For example, Park optimized the acceleration in order to maximize the efficiency [11]. Pellicciari et al., as well as Wigström et al., proposed energy-efficient trajectories by means of constant and variable time-scaling methods $[12,13]$. Bobrow et al. focused 
on the minimization of the time required when path and actuation torque limitations are prescribed [14].

This is where velocity scheduling becomes interesting, as it does not change the path to follow. Instead, the robot is slowed down/speeded up at certain points of the trajectory to minimize the maximum torque required from the actuator. Therefore, with the same actuators, the smallest achievable cycle time can be reduced without any modification to the prescribed path. In this paper, a heuristic algorithm, aimed at reducing the maximum torque required to perform the industry-standard Adept test-cycle, is proposed. It can, however, be applied to any other trajectory. This approach differs from the one reported elsewhere by the same authors, in which a limited number of points were selected [15]. The robot used to evaluate the performance of the algorithm is the Peppermill Carrier (PMC), an isostatic SMG actuated with two cylindrical drives (C-drives), developed at McGill University. The balance of the paper is organized as follows: In Section 2, the dynamics model of the PMC is recalled, while in Section 3, the Adept test-cycle is described. In Section 4, the velocity scheduling algorithm is introduced, while in Sections 5 and 6, results and their discussion are included.

\section{Kinematics and Dynamics}

The model that describes the relations between the various parameters and the motion variables of the PMC, in both the Cartesian and the joint domains, is needed to conduct the necessary simulation work to validate the algorithm proposed in the sequel. This model was reported in a previous paper [16]; it is recalled here for the sake of completeness. The isostatic CRRHHRRC architecture of the PMC is illustrated in Fig. 1. It consists of two C-drives, two limbs made of one arm and one forearm, as well as the Peppermill, to which the moving plate is attached. The C-drives are differential mechanisms capable of generating translational and rotational motion of the cylindrical type independently, with the former along a direction parallel to the axis of the latter. The synchronized rotation of two nuts, driven by two parallel screws, one right-, one lefthand, attached to a DC motor, makes the controlled cylindrical motion possible. The rotation and translation of the nuts are transferred to a strain-wave-gear (SWG) transmission mounted on a third parallel axis by a belt-pulley transmission [17]. The total gear-reduction ratio due to the latter and the SWG is represented by the constant $G$.

The actuation of the C-drives, defined by the variables $u_{i}$ and $\theta_{i}$, where $i=\{1,2\}$, is arrayed in the vector $\mathbf{d}$ for the translation and the rotation of the $i$ th C-drive output, respectively. Vector $\mathbf{d}$ is mapped by the $4 \times 4$ Jacobian matrix $\mathbf{J}_{C}$ into the joint variables $\boldsymbol{\psi} \equiv\left[\begin{array}{llll}\psi_{1 L} & \psi_{1 R} & \psi_{2 L} & \psi_{2 R}\end{array}\right]^{T}$ :

$$
\boldsymbol{\psi}=\mathbf{J}_{C} \mathbf{d}
$$

with

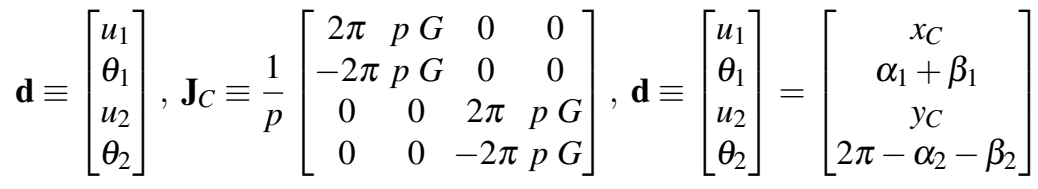




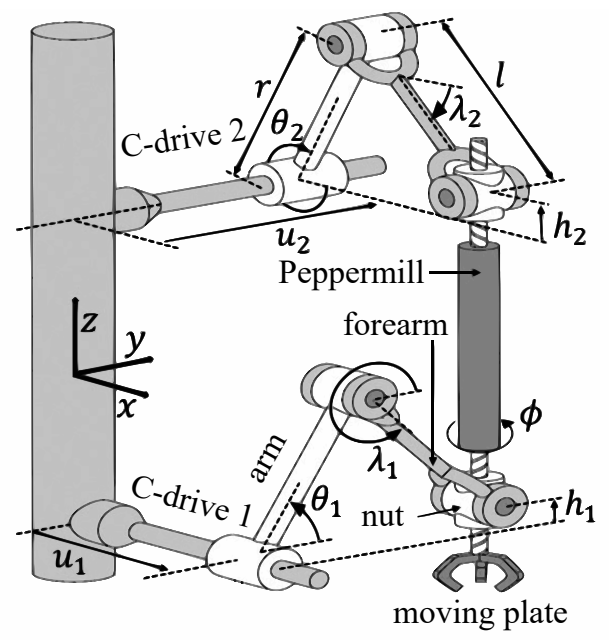

Fig. 1: PMC schematic view.

The indices $1 L, 1 R, 2 L$, and $2 R$, respectively, stand for the left-hand and righthand screws of the first and the second C-drives. The MP pose is defined by the fourdimensional vector $\mathbf{x} \equiv\left[x_{C}, y_{C}, z_{C}, \phi\right]^{T}$. Variables $\alpha_{i}$ and $\beta_{i}$, appearing in d, eq.(1b), are to be computed with the expressions:

$$
\begin{gathered}
\alpha_{1}=\arctan \left(\frac{h_{1}}{y_{C}}\right), \quad \alpha_{2}=\arctan \left(\frac{h_{2}}{x_{C}}\right), \quad \beta_{i}=\arccos \left(\frac{r^{2}-l^{2}+k_{i}^{2}}{2 r k_{i}}\right) \\
k_{1}=\sqrt{y_{C}^{2}+h_{1}^{2}}, \quad k_{2}=\sqrt{x_{C}^{2}+h_{2}^{2}}, \quad h_{i}=z_{C}+(-1)^{i} \frac{q \phi}{2 \pi}, \quad i=1,2
\end{gathered}
$$

The pitch of the right- and the left-hand screws of the C-drives are, respectively, $p$ and $-p$, while the pitch of the right- and the left-hand screws of the Peppermill are, respectively, $q$ and $-q$. The other geometric parameters are indicated in Fig. 1.

The mathematical model used to compute the motor torques $\tau$ was obtained with the method of the natural orthogonal complement (NOC) [18]. The PMC is made up of 11 rigid bodies: four screws, two arms, two forearms, two nuts, and the Peppermill. The torque vector is computed as

$$
\tau=\tau_{d}+\tau_{s}
$$

where

$$
\tau_{d} \equiv \mathbf{I} \ddot{\boldsymbol{\psi}}+\mathbf{C} \dot{\boldsymbol{\psi}}, \quad \boldsymbol{\tau}_{s} \equiv-\boldsymbol{\gamma}-\boldsymbol{\eta}
$$

with

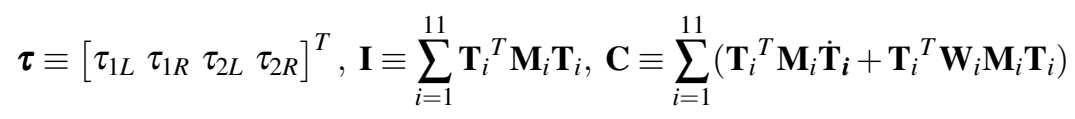




$$
\boldsymbol{\gamma}+\boldsymbol{\eta} \equiv \sum_{i=1}^{11} \mathbf{T}_{i}^{T}\left(\mathbf{w}_{i}^{G}+\mathbf{w}_{i}^{E}\right), \mathbf{M}_{i} \equiv\left[\begin{array}{cc}
\mathbf{I}_{i} & \mathbf{O} \\
\mathbf{O} & m_{i} \mathbf{1}
\end{array}\right], \mathbf{W}_{i} \equiv\left[\begin{array}{cc}
\boldsymbol{\Omega}_{i} & \mathbf{O} \\
\mathbf{O} & \mathbf{O}
\end{array}\right], \mathbf{T}_{i} \equiv \frac{\partial \mathbf{t}_{i}}{\partial \dot{\boldsymbol{\psi}}}
$$

where $\mathbf{1}$ and $\mathbf{O}$ denote the $3 \times 3$ identity and zero matrices. The angular-velocity matrix $\boldsymbol{\Omega}_{i}$ is used to compute the angular-velocity dyad $\mathbf{W}_{i}$ of the $i$ th body. The former is the cross-product matrix $(\mathrm{CPM})^{1}$ of the angular-velocity vector $\boldsymbol{\omega}_{i}$. The twist-shaping matrix of the $i$ th body, $\mathbf{T}_{i}$, maps the vector array of the motors speeds, $\dot{\psi}$, into the twist vector $\mathbf{t}_{i}$ of the $i$ th body. Furthermore, $\mathbf{I}_{i}$ and $\mathbf{M}_{i}$ are, respectively, the inertia tensor and the inertia dyad at the center of mass (c.o.m.) of the $i$ th body. Finally, $\mathbf{w}_{i}^{G}$ and $\mathbf{w}_{i}^{E}$ are the gravitational and external wrenches exerted on the $i$ th body, which are used to compute $\boldsymbol{\gamma}$ and $\boldsymbol{\eta}$, their corresponding generalized-force vectors. The twist $\mathbf{t}_{i}$ of and the wrench $\mathbf{w}_{i}$ exerted on the $i$ th body are evaluated at the body c.o.m. and defined as

$$
\mathbf{t}_{i} \equiv\left[\begin{array}{c}
\boldsymbol{\omega}_{i} \\
\mathbf{v}_{i}
\end{array}\right], \quad \mathbf{w}_{i} \equiv\left[\begin{array}{c}
\mathbf{n}_{i} \\
\mathbf{f}_{i}
\end{array}\right]
$$

where $\mathbf{v}_{i}$ and $\boldsymbol{\omega}_{i}$ represent, respectively, the velocity of the $i$ th body c.o.m., and its angular velocity. The vectors $\mathbf{n}_{i}$ and $\mathbf{f}_{i}$ are the moment and the force acting on the $i$ th body, the former about, the latter at the body c.o.m.

\section{The Adept Test-Cycle}

The Adept test-cycle [1] is the trajectory used in this paper to evaluate the potential of the algorithm proposed. This cycle is an industry standard task used by the robotics community to evaluate pick-and-place robots. It starts with a vertical upward translation of $25 \mathrm{~mm}$, followed by a horizontal 300-mm translation with a concurrent $180^{\circ}$ rotation about the vertical axis, then a vertical downward $25-\mathrm{mm}$ translation; finally, a symmetric motion back to the original pose of the robot, closes the cycle. The trajectory was smoothed to avoid velocity and acceleration discontinuities [19].

The maximum torque reached at any point of the trajectory, regardless of the actuator, is obtained as a function of the operation frequency. The results are illustrated in Fig. 2. The maximum torque as a function of time is is described below:

$$
\tau_{\max }(t)=\max \left\{\left|\tau_{1 L}\right|,\left|\tau_{1 R}\right|,\left|\tau_{2 L}\right|,\left|\tau_{2 R}\right|\right\}
$$

The maximum torque increases with the square of the frequency, thereby showing the need of an adequate velocity scheduling. The trajectory of the PMC is symmetric with respect to the middle point of the trajectory, which is expected, considering that the PMC is stationary at this point and follows the same path backwards with the same duration. To reduce the torque peaks, a symmetric velocity scheduling program might as well be applied.

\section{Velocity Scheduling}

An iterative procedure is used to gradually reduce the peaks observed. Indeed, considering the variation of $\tau_{\max }(t)$, as shown in Fig. 2, it would be challenging to predict

\footnotetext{
${ }^{1}$ The CPM of $\boldsymbol{\omega}_{i}$ is defined as the partial derivative of $\boldsymbol{\omega}_{i} \times \mathbf{y}$ with respect to $\mathbf{y}, \forall \mathbf{y} \in \mathbb{R}^{3}$.
} 

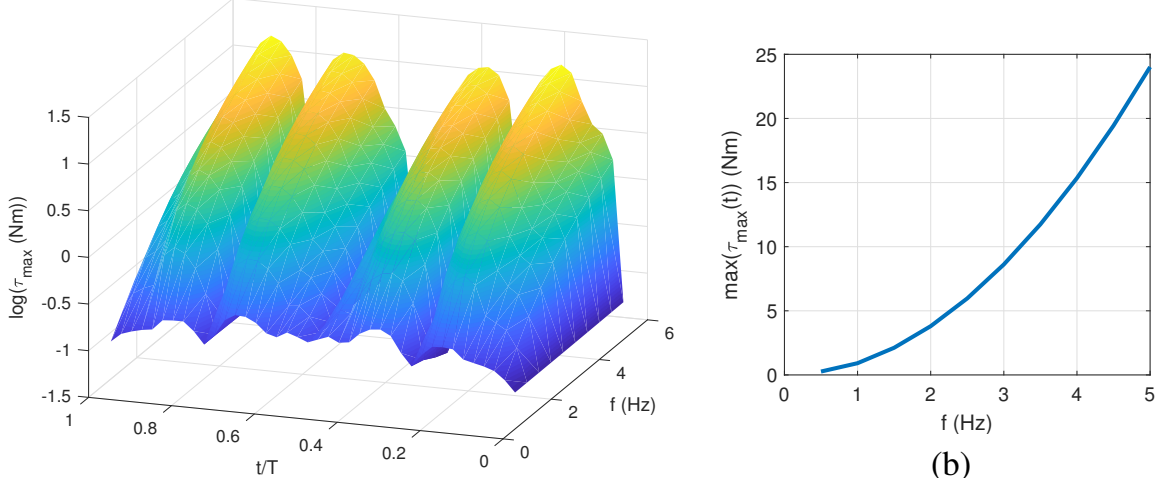

(b)

(a)

Fig. 2: Maximum torque reached: (a) along the trajectory as a function of time; (b) for the whole trajectory.

the effect of a drastic change in the velocity scheduling. Therefore, with a time-scaling function with variable parameters, the maximum torque would be minimized. Torques at the actuators for the test-cycle, produced at $1 \mathrm{~Hz}$, are shown in Fig. 3.

A simple means to reduce the torque peaks is to compute the mean value $\bar{\tau}$ of $\tau_{\text {max }}(t)$, and slowing down/speeding up the robot whenever $\left(\tau_{\max }(t)-\bar{\tau}\right)$ changes sign. This difference is multiplied by a factor $C$, as yet to be determined:

$$
K_{s c h}=C\left(\tau_{\max }(t)-\bar{\tau}\right)+1
$$

The area beneath the above function is always equal to the cycle time, which ensures that the time required to follow the trajectory remains unchanged. Because the optimum value of $C$ cannot be predicted, a range of values must be tested. Indeed, too large a magnitude could create new peaks where there were none, while too small would not significantly cut down the maximum value of $\tau_{\text {max }}(t)$. Moreover, the time-scaling function proposed in eq.(5) must also be smoothed to avoid amplifying existing torque peaks and creating new ones. To smooth this function, an average filter is used, with $N$ neighboring entries. Again, different values of $N$ are tested to obtain the best result.

The inverse-dynamics analysis (IDA) conducted in Section 2 is first used to obtain $\tau_{\max }(t)$. From the latter, the value of $\bar{\tau}$ can be calculated. With a predetermined set of $\{C\}$ and $\{N\}$ values, several smoothed time-scaling functions are obtained. Each of them is applied to the trajectory, and the IDA is again used to find the maximum value of $\tau_{\text {max }}(t)$. The velocity schedule minimizing $\max \left(\tau_{\max }(t)\right)$ is chosen. Afterwards, the whole algorithm is to be repeated with the new scheduled trajectory. The process stops once the resulting maximum values of $\tau_{\max }$ converge toward a minimum. 


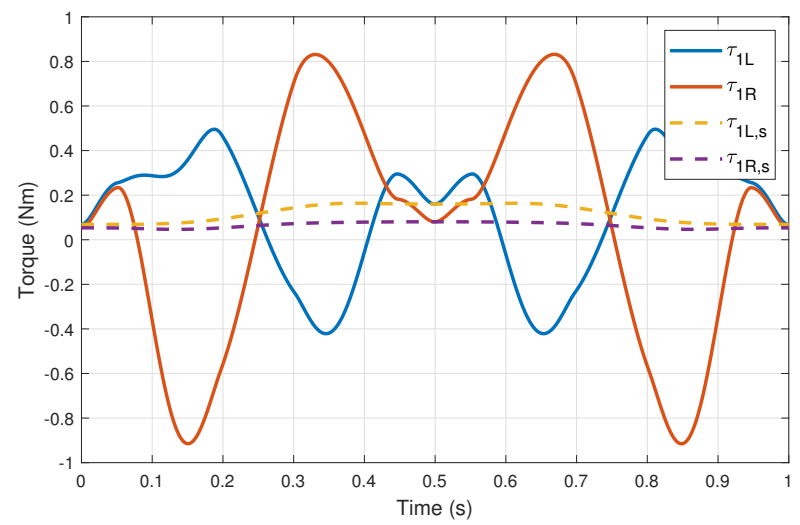

Fig. 3: Static $\left(\tau_{i, s}\right)$ and total $\left(\tau_{i}\right)$ torques at each actuator with a trajectory performed at $1 \mathrm{~Hz}$.

\section{Simulation Results}

With the dynamics model and the velocity-scheduling algorithm detailed above, it is possible to reduce significantly the torque peaks previously observed with the initial trajectory. As shown in Fig. 4, a reduction of more than $40 \%$ of the maximum torque for the same path at a frequency of $1 \mathrm{~Hz}$ is obtained.

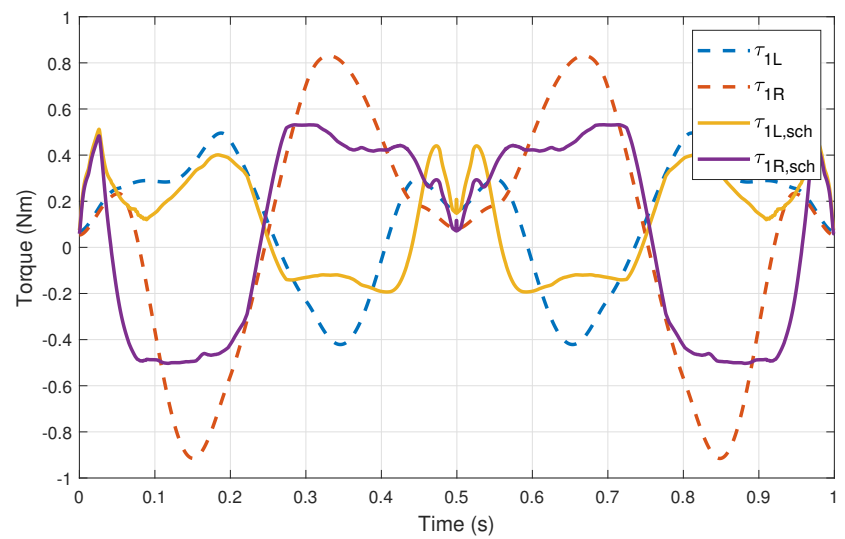

Fig. 4: Original and scheduled trajectories with a cycle time of $1 \mathrm{~s}$.

The successive time-scaling functions applied to the trajectory are shown in Fig. 5. For the record, 11 iterations were required to converge toward a minimum value of $\max \left\{\tau_{\max }(t)\right\}$. As can be appreciated in Fig. 4, one particular point in the trajectory did 
not notably change. Indeed, around $0.25 \mathrm{~s}$, there is a local minimum that was mostly unaffected by the algorithm. This is caused by the nature of this point, where the actuator generating the maximum torque is not the same before and thereafter. Moreover, while the robot remains in motion, the time-varying torque is significantly low at this point, meaning that the total torque at the same point is mostly caused by gravity alone.

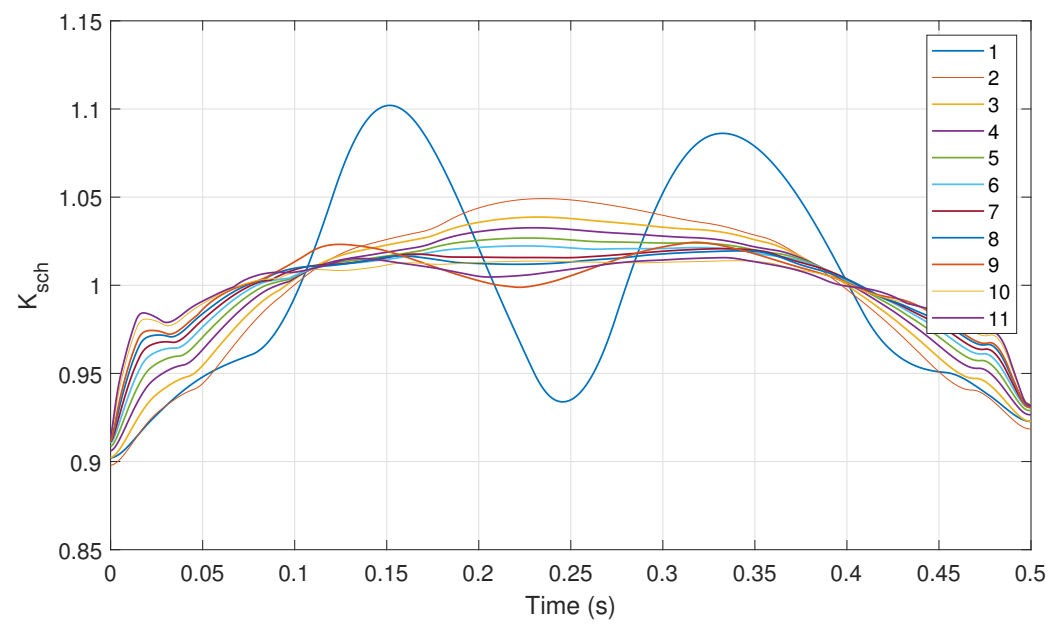

Fig. 5: Successive time-scaling functions applied to the $1 \mathrm{~Hz}$ trajectory (only the first half shown, as it is symmetric).

The pose of the MP as a function of time, for the original and the scheduled velocities, is illustrated in Fig. 6. As can be seen, the modification induced by the velocityscheduling algorithm had a relatively minor impact on the pose of the MP as a function of time. It should be recalled that the path and cycle time remain the same in both cases, as the same velocity scheduling is applied to the four actuators.

\section{Discussion}

The proposed velocity scheduling algorithm was applied to different cycle time values to assess its efficiency. As detailed in Table 1, similar results were obtained regardless of the operating frequency. While the maximum torque increases significantly, the algorithm was still able to keep it even smaller by more than $40 \%$. When the cycle time is higher than 1 second, its impact is lower, as the effect of gravity overcomes the inertia forces of the system due to its motion.

The proposed algorithm can be used with any pick-and-place robot, as it only relies on the torque required along the trajectory. Because the model used in conjunction with the algorithm does not exactly reflects the actual robot, discrepancies are to be expected. 

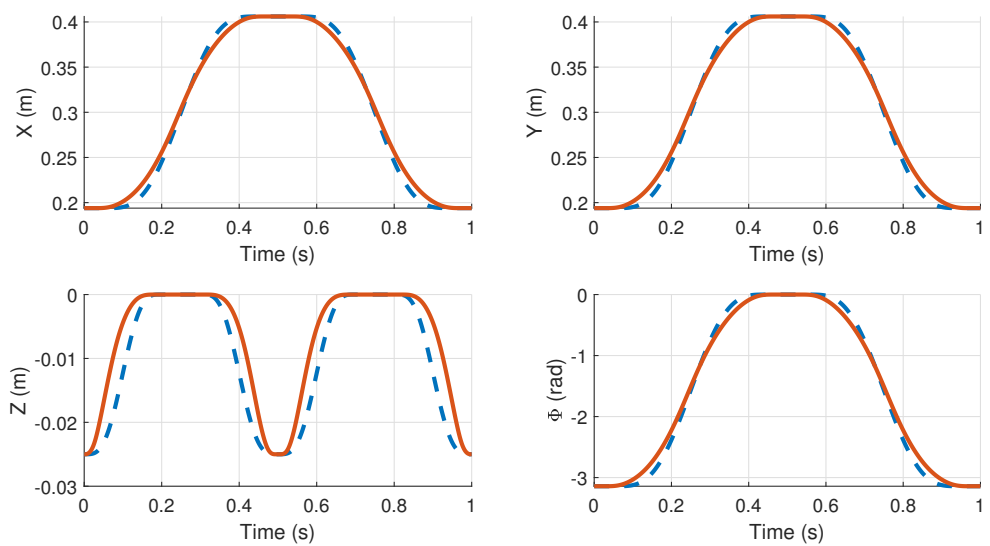

Fig. 6: Pose of the MP, dashed curves for the original trajectory, solid curves for the scheduled trajectory.

Table 1: Efficiency of the velocity scheduling algorithm with different cycle times.

\begin{tabular}{llll}
\hline \hline Frequency $(\mathrm{Hz})$ & Original $\tau_{\max }(\mathrm{Nm})$ & Scheduled $\tau_{\max }(\mathrm{Nm})$ & Reduction \\
\hline 1 & 0.9148 & 0.5324 & $41.80 \%$ \\
2 & 3.800 & 2.009 & $47.13 \%$ \\
3 & 8.610 & 4.578 & $46.83 \%$ \\
4 & 15.34 & 8.622 & $43.79 \%$ \\
5 & 24.00 & 12.62 & $47.42 \%$ \\
\hline \hline
\end{tabular}

However, peaks and lows should occur relatively at the same points with both the model and the prototype.

\section{Conclusions}

The speed and trajectory-tracking accuracy of pick-and-place robots are two crucial elements when it comes to their fast operations. However, increasing one beyond a certain point commonly comes at the expense of the other. In this paper, an algorithm was proposed to reduce the torque requirements to produce the Adept test-cycle without changing either the path or the cycle time. To reach this goal, the velocity scheduling was modified with a heuristic algorithm that reduced the maximum torque by more than $40 \%$, regardless of the operating frequency. Simulation tests were conducted with a model of the Peppermill Carrier, an isostatic Schönflies-motion generator actuated by novel cylindrical drives. The results validated the effectiveness of the proposed algorithm. 


\section{References}

1. Nabat, V., Rodriguez, M., Company, O., Krut, S., Pierrot, F.: Par4: Very high speed parallel robot for pick-and-place. In: Proceedings of the IEEE/RSJ International Conference on Intelligent Robots and Systems. Edmonton, Canada (2005).

2. Makino, H., Kato, A., Yamazaki, Y.: Research and commercialization of SCARA robot-the case of industry-university joint research and development. International Journal of Automation Technology. 1, 61-67 (2007).

3. Brogårdh, T.: Device for Relative Movement of Two Elements. U.S. Patent No. 6,301,988 (2001).

4. Angeles, J., Morozov, A.: Four-Degree-of-Freedom Parallel Manipulator for Producing Schönflies Motions. U.S. Patent No. 7,127,962 (2006).

5. Gallardo-Alvarado, J., Abedinnasab, M.H.,Lichtblau, D.: Simplified Kinematics for a Parallel Manipulator Generator of the Schönflies Motion. Journal of Mechanisms and Robotics. 8(6), 061020 (2016).

6. Corbel, D., Gouttefarde, M., Pierrot, F.: Actuation redundancy as a way to improve the acceleration capabilities of 3T and 3T1R pick-and-place parallel manipulators. Journal of Mechanisms and Robotics. 2(4), 041002 (2010).

7. Altuzarra, O., Zubizarreta, A., Cabanes, I., Pinto, C.: Dynamics of a four degrees-of-freedom parallel manipulator with parallelogram joints. Mechatronics, 19(8), 1269-1279 (2009).

8. Zhang, N., Shang, W. Dynamic trajectory planning of a 3-DOF under-constrained cable-driven parallel robot. Mechanism and Machine Theory. 98, 21-35 (2016).

9. Gosselin, C., Foucault, S.: Dynamic point-to-point trajectory planning of a two-DOF cablesuspended parallel robot. IEEE Transactions on Robotics. 30(3), 728-736 (2014).

10. Jiang, X., Gosselin, C.: Dynamic point-to-point trajectory planning of a three-DOF cablesuspended parallel robot. IEEE Transactions on Robotics. 32(6), 1550-1557 (2016).

11. Park, J.S.: Motion profile planning of repetitive point-to-point control for maximum energy conversion efficiency under acceleration conditions. Mechatronics. 6(6), 649-663 (1996).

12. Pellicciari, M., Berselli, G., Leali, F., Vergnano, A.: A method for reducing the energy consumption of pick-and-place industrial robots. Mechatronics. 23, 326-334 (2013).

13. Wigström, O., Lennartson, B., Vergnano, A.: High-Level Scheduling of Energy Optimal Trajectories. IEEE Transactions on Automation Science and Engineering. 10(1), 57-64 (2013).

14. Bobrow, J.E., Dubowsky, S., Gibson, J.S.: Time-optimal control of robotic manipulators along specified paths. The International Journal of Robotics Research. 4(3), 3-17 (1985).

15. Karimi Eskandary, P., Belzile, B., Angeles, J.: Trajectory-Planning and Normalized-Variable Control for Parallel Pick-and-Place Robots. ASME Journal of Mechanisms and Robotics. In press, 1-15 (2018).

16. Karimi Eskandary, P., Angeles, J.: The dynamics of a parallel Schöflies-motion generator. Mechanism and Machine Theory. 119, 119-129 (2018).

17. Karimi Eskandary, P., Angeles, J.: The translating $\Pi$-joint: Design and applications. Mechanism and Machine Theory. 122, 361370 (2018).

18. Angeles, J., Lee, S.:The formulation of dynamical equations of holonomic mechanical systems using a natural orthogonal complement. ASME J. of Applied Mechanics, 55(1), 243-244 (1988).

19. Gauthier, J.F., Angeles, J., Nokleby, S.B.: Optimization of a test trajectory for SCARA systems. In: Lenarčič, Thomas, F. (eds.) On Advances in Robot Kinematics. pp. 225-234. Springer, Amsterdam (2008). 